\title{
Pratiques
}

Linguistique, littérature, didactique

141-142| 2009

La synonymie

\section{Synonymes : petite bibliographie d'études descriptives}

\section{Patrick Dendale}

\section{(2) OpenEdition}

1 Journals

Édition électronique

URL : https://journals.openedition.org/pratiques/1371

DOI : 10.4000/pratiques. 1371

ISSN : 2425-2042

Éditeur

Centre de recherche sur les médiations (CREM)

\section{Édition imprimée}

Date de publication : 15 juin 2009

Pagination : 233-248

\section{Référence électronique}

Patrick Dendale, «Synonymes : petite bibliographie d'études descriptives », Pratiques [En ligne], 141-142 | 2009, mis en ligne le 13 juin 2014, consulté le 11 mars 2023. URL : http://

journals.openedition.org/pratiques/1371; DOI : https://doi.org/10.4000/pratiques.1371

Tous droits réservés 


\title{
Synonymes : petite bibliographie d'études descriptives
}

\author{
Patrick Dendale
}

Université d'Anvers (Center for Grammar, Cognition and Typology \& Antwerp Center for Pragmatics) \& CELTED (Metz)

\section{Introduction}

1. Dans cette bibliographie restreinte sont rassemblées des références d'études portant sur des mots sémantiquement apparentés du français (français moderne, médiéval et classique). Il s'agit d'une bibliographie d'études descriptives, empiriques de synonymes, non d'une bibliographie d'études théoriques sur le phénomène ou la notion de synonymie. N'y ont été retenues que les études dans lesquelles sont décrites au moins deux expressions ${ }^{(1)}$. Tantôt ces expressions sont explicitement mises en rapport comme (quasi-/pseudo-)synonymes ( $c f$. des titres comme "Les synonymes an-année : problème trivial insoluble? ", "Note sur la pseudo-synonymie de Il s'agit et Il est question", "Quand même et tout de même : vrais ou faux synonymes? ", "Au contraire, par contre, en revanche: une évaluation de la synonymie ») ; tantôt les expressions en question sont décrites séparément, les descriptions individuelles mises en parallèle ou juxtaposées (leur comparaison est alors laissée au lecteur).

2. La sélection des références a été réalisée à partir de la bibliographie Lexicales, qui est librement accessible sur Internet ${ }^{(2)}$ et qui contient actuellement quelque 2000 références d'études sémantiques, syntaxiques et pragmatiques - articles, monographies et thèses - portant sur des unités lexicales (et grammaticales) françaises.

(1) Le lecteur qui désire une information plus complète sur telle ou telle unité précise (par exemple sur quelques, plusieurs,...) peut se composer une bibliographie thématique sur le site mentionné (partie «Bibliographie thématique»).

(2) Voir http ://webh01.ua.ac.be/lexico/. Une fois par an la bibliographie est mise à jour et augmentée de plusieurs centaines de références. 


\section{Quelles sont les références retenues ici ?}

3.1. Il s'agit d'abord d'études portant sur des unités synonymes du lexique, simples (an / année ; tenter / essayer ; seulement / uniquement ; décade / décennie), ou complexes (à l'aide de / au moyen de; au sujet de / à propos de; il semble que / il paraît que) ou des unités sémantiquement proches appartenant à l'un ou l'autre sous-système grammatical de la langue (pas / point ; ne / non ; tu / vous ; je / moi / me ; son / le sien ; que / quoi, quelques / plusieurs / certains / divers). Toutes ces paires ou séries de mots partagent certains traits sémantiques importants, mais les mots composants se distinguent entre eux par d'autres traits plus ou moins nombreux (bout et brin par exemple sont tous les deux « des morceaux d'un élément concret » (Normand 1997 :108), mais se distinguent par l'objet dont on désigne un morceau).

Très souvent - c'est là une caractéristique connue de la relation de synonymie - les différentes unités « synonymes » n'ont de définitions sémantiques parallèles que pour certains de leurs emplois : par exemple sur / à dans le tableau est sur le / au mur; à / avec dans à la hache / avec la hache; en / de dans pullover en laine / de laine; autre / second dans ajouter un autre couvert / un second couvert; où / que dans le jour où / le jour que ; à / par pour l'indication de l'agent d'un verbe passif.

La parenté sémantique entre ces unités peut se manifester dans des distributions comparables (il / ça pleut, aller au / chez le coiffeur, lui / y), sans qu'on puisse parler de définitions sémantiques identiques (Jean arriva. Alors / Puis le concert commença).

3.2. Ont été reprises ici, deuxièmement, des études portant sur des unités construites. Deux types de travaux ont été sélectionnés : ceux qui comparent des unités du lexique à des unités construites (voiturette / petite voiture, décider / prendre la décision, par terre / sur le sol, peu / un peu); ceux qui étudient des structures, voire des constructions sémantiquement équivalentes (à mon avis / pour moi / selon moi ; rien moins que / rien de moins que; qu'est-ce que c'est? / c'est quoi ? ; il est linguiste / c'est un linguiste ; ton neveu, il est orgueilleux / ton neveu, c'est un orgueilleux).

3.3. N'ont pas été reprises les études portant sur des variantes phonologiques d'une même unité (on / l'on) ou sur des variantes dialectales d'un même mot (polailli, poule, poulaille).

4. Pour ce qui est du contenu de la description, les études retenues portent soit sur les ressemblances et les différences de sens et/ou de construction entre les unités, (connaître, contrairement à savoir, refuse les propositions complétive et infinitive), soit sur leurs potentialités d'insertion dans le discours (les enchaînements à des énoncés contenant peu ou un peu ou puis / alors ne sont pas les mêmes). 


\section{La bibliographie}

La liste des références, classée alphabétiquement par auteur, se présente sous forme d'un tableau à deux colonnes : la première colonne contient la référence, la seconde les unités ou expressions étudiées.

ANSCOMBre, Jean-Claude, 1990, «L'opposition longtemps / longuelongtemps / ment : durée objective et durée subjective ", Langue française, 88, longuement pp. 90-117.

ANSCOMBRE, Jean-Claude, 1996, «L'opposition surtout / particulièrement et la structuration discursive », in Muller, Claude (éd.), 1996, Dépendance et intégration syntaxique. Subordination, coordination, connexion, Tübingen, Niemeyer, pp. 245-256.

Anscombre, Jean-Claude, 2006, «Périr, ce n'est pas mourir un peu. Sur une structuration aspectuelle et temporelle locale du lexique verbal», in Riegel, Martin e.a. (éds), 2006, Aux carrefours du sens. Hommages offerts à Georges Kleiber pour son 60e anniversaire, Louvain, Peeters, pp. 39-51.

ANSCOMBRe, Jean-Claude, 2006, «Tout, n'importe quel, chaque : quelques remarques ", in Corblin, Francis, Ferrando, Sylvie \& Kupferman, Lucien (éds), 2006, Indéfini et prédication, Paris, PUPS, pp. 431-448.

Anscombre, Jean-Claude, 2006, « Les locutions quant à, pour ce qui est de, en ce qui concerne : chronique d'un discours annoncé », Modèles Linguistiques, 27, 2, pp. 155-169.

ARVEILlER, Raymond, 1978, "Airelle, myrtille ", in Lafont, Robert e.a. (éds), 1978, Mélanges de philologie romane offerts à Charles Camproux, tome 2, Montpellier, CE0, pp. 809-820.

BARRY, Alpha Ousmane, 2001, «Je vous vouvoie et tu me tutoies ! reflet de la diversité des cultures francophones de Guinée. Enjeux didactiques des théories du texte dans le cadre des enseignements / apprentissages bilingues », Nouveaux cabiers d'allemand, 19, 2, pp. 339-348.

BeAUliEU-MaSSON, Anne, 2004, « Les connecteurs face à l'énonciation : les anaphores énonciatives existent-elles ? », in Rossari, Corinne (éd.), 2004, Autour des connecteurs. Réflexions surl'énonciation et la portée, Berne, Peter Lang, pp. 123-156.

BELTAÏEF, Lilia, 2004, "Quand même et tout de même : vrais ou faux synonymes ? », Revue de sémantique et pragmatique, 15/16, pp. 253265.

Ben HAMAD, Leila, 2004, «Pendant et durant sont-ils vraiment synonytout / n'importe quel / chaque quant à / pour ce qui est de / en ce qui concerne airelle / myrtille mes? », Revue de sémantique et pragmatique, 15/16, pp. 221-234.

Berthonneau, Anne-Marie \& CADIOT, Pierre, 1991, «Pendant et pour, variations sur la durée et donation de la référence : prépositions, représentations, référence ", Langue française, 91, pp. 102-124.

Berthonneau, Anne-Marie, 1992, "Comment depuis et ily a parlent-ils tu / vous du temps? », in Flament-Boistrancourt, Danièle (éd.), 1992, Théories, données et pratiques en français langue étrangère, Lille, Presses universitaires de Lille, pp. 61-93. 
BERTHONNEAU, Anne-Marie, 1999, « ̀̀ propos de dedans et de ses relations avec dans ", Revue de sémantique et pragmatique, 6, pp. 13-41.

Besse, Henri, 1973, "Parce que et puisque », Voix et Images du Crédif, 18 , pp. 26-32.

BLANCHE-BENVENISTE, Claire, 1997, «À propos de Qu'est-ce que c'est et C'est quoi ", Recherches sur le français parlé, 14, 1, pp. 127-146.

BORILLO, Andrée, 2004, "Les "adverbes d'opinion forte" selon moi, à mes yeux, à mon avis,... : point de vue subjectif et effet d'atténuation ", Langue française, 142, pp. 31-40. dedans / dans

parce que / puisque

qu'est-ce que c'est / c'est quoi selon moi / à mes yeux / à mon avis

BouRDIN, Philippe, 1986, «Sembler et paraître ou les deux visages de sembler / paraître l'apparence », Semantikos, 10, 1-2, pp. 45-67.

BouTET, Josiane, 1988, « La concurrence de on et de $i$ en français parlé », on / i(ls) Linx , 1, 18, pp. 47-66.

Bryan, Anne-Marie, 1971-1972, « Le tu et le vous », French Review, 45, tu / vous pp. 1007-1010.

CADIOT, Pierre, 1989, « Les alternances entre les prépositions pour et de : pour / de de la grammaire à la cognition ", in Lorenzo, Ramón (éd.), 1997, Actas do XIX Congreso Internacional de Lingüística e Filoloxía Románicas, Coruña, Pedro Barrié de la Maza, pp. 363-376.

CADIOT, Pierre, 1991, " A la hache ou avec la hache ? Représentation mentale, expérience située et donation du référent », Langue française, 91, pp. 7-23.

CADIOT, Pierre, 1993, «De et deux de ses concurrents : avec et $\grave{a}$ »,Langages, 110, pp. 68-106.

CAmus, Rémi, 1992, "De nouveau / à nouveau : du nouveau ", L'information grammaticale, 55, pp. 17-22.

CAPT-ARTAUD, Marie-Claire, 1993, "Redouter, craindre, avoir peur... ou la plus belle pièce de l'héritage rhétorique », Cabiers Ferdinand de Saussure, 47, pp. 23-36.

$\grave{a} /$ avec

Cartmill, Constance, 2004, "Lorsqu'un On vaut un Je : Emplois du pronom indéfini chez Mme de Sévigné ", Neophilologus, 88, 2, pp. 203-217.

Charles, Pol, 1998, « Les tournures rien moins que et rien de moins que », in Ruffino, Giovanni (éd.), 1998, Atti del XXI Congresso Inter- rien de moins que nazionale di Linguistica e Filologia Romanza. Vol. 2, Morfologia e sintassi delle lingue romanze, Palermo/Tübingen, Niemeyer, pp. 155-174.

Charolles, Michel \& Lamiror, Béatrice, 2007, « Du lexique à la grammaire : seulement, simplement, uniquement ", Cahiers de lexicologie, 90, 1, pp. 93-116.

CHRISTIAN, Rohrer, 1981, «Quelques remarques sur les différences entre à partir de, depuis, dans une heure, une heure plus tard ", in Schwarze, Christophe (éd.), 1981, Analyse des prépositions, Tübingen, Niemeyer, pp. 158-170.

de / avec / à

de nouveau / à nouveau redouter / craindre / avoir peur on / je rien moins que / .

.

seulement /
simplement /
uniquement
à partir de /
dans une heure /
depuis /
une heure plus tard


CoJoCARIU, Corina, 2004, « Les adverbes de validation. Quelques hypothèses ", in Rossari, Corinne (éd.), 2004, Autour des connecteurs. Réflexions sur l'énonciation et la portée, Berne, Lang, pp. 183-214.

COJOCARIU, Corina, 2005, «Nécessairement et forcément : deux adverbes synonymes? », Revue romane, 40, 1, pp. 23-46.

ColTIER, Danielle \& DENDALE, Patrick, 2004, « La modalisation du discours de soi : éléments de description sémantique des expressionspour moi, selon moi, et à mon avis », Langue française, 142, pp. 41-57.

CORNILLAC, Guy, 2004, « Relation entre syntaxe interne et syntaxe externe : voiturette et petite voiture ", Modèles linguistiques, 25, 1-2, pp. 139-144.

CORTÈs, Colette, 1997, «Décider, prendre une décision de : du verbe à la locution verbale », in Fiala, Pierre, Lafon, Pierre \& Piguet, MarieFrance (éds), 1997, La locution : entre lexique, syntaxe et pragmatique, Paris, Klincksiek, pp. 19-36.

DagenaIs, Louise, 1985, « Le problème de la description sémantique en lexicographie. Les vocables français : permettre, défendre, autoriser, interdire,... », Cabiers de lexicologie, 46, pp. 57-107. en effet /

effectivement / certes / oui / soit / d'accord nécessairement / forcément pour moi / selon moi / à mon avis voiturette / petite voiture décider / prendre une décision permettre / défendre / autoriser / interdire

DAMAR, Marie-Ève, 2006, «Rien moins que, rien de moins que : un problème de grammaire revisité », Travaux de linguistique, 53, rien de moins que pp. 117-133.

DANELL, Karl-Johan, 1990, « Les synonymes an-année : problème trivial insoluble? », in Halmøy, Odile, Halvorsen, Arne \& Lorentzen, Lise (éds), 1990, Actes du $11^{e}$ congrès des romanistes scandinaves, Trondheim, Université de Trondheim, pp. 1-25.

DANELL, Karl-Johan, 1995, Le phénomène de concurrence en français an / année / jour / moderne. Réflexions à partir de an-année, jour-journée, paraître- journée / paraître / apparaître et d'autres, Uppsala, Swedisch Science Press apparaître

DANJOU-FLAUX, Nelly, 1980, «Au contraire, par contre, en revanche : une évaluation de la synonymie ", Bulletin du centre d'analyse du discours, 4, pp. 123-146.

au contraire / par contre / en revanche

Danjou-Flaux, Nelly, 1982, «Réellement et en réalité : données lexicographiques et description sémantique », Lexique, 1, pp. 105-150.

réellement / en réalité / dans la réalité

Danjou-Flaux, Nelly, 1984, «Au contraire, par contre, en revanche», Lingvisticae Investigationes, 8, pp. 75-94.

au contraire / par contre / en revanche

DANON-BOILEAU, Laurent \& MOREL, Mary-Annick, 1992, « Opérations énonciatives et valeurs argumentatives : étude de trois marqueurs concessifs (bien que, même si, encore que) ", in Perrin, Isabelle (éd.), 1992, Approches énonciatives de l'énoncé complexe, Louvain-Paris, Peeters, pp. 7-20.

De Penanros, Hélène, 2002, «Lors de, au moment où, ou à l'occasion de », Cahiers Chronos, 10, pp. 201-215. même si / encore que lors de / au moment où / à l'occasion de bien que / 
DE VogüÉ, Sarah, 1992, « Aux frontières des domaines notionnels : bien bien que / que, quoique et encore que ", L'information grammaticale, 55, quoique / pp. 23-27.

encore que

DeBaISIEUX, Jeanne-Marie, 2001, « Contraintes syntaxiques et discursives quant à / des emplois de quant à et en ce qui concerne ", Cabiers de praxéma- en ce qui concerne tique, 37, pp. 125-146.

DELABRE, Michel, 1984, « Syntaxe de ainsi que et de de même que en franainsi que / çais contemporain ", L'information grammaticale, 23, pp. 11-17. de même que

DelBEY, Annie, 1988, «Les connecteurs car-puis - puis que et la justification en ancien français », Revue de linguistique romane, 52, 207208, pp. 397-419.

Dendale, Patrick \& DE Mulder, Walter, 1998, « Contre et sur : du spatial contre / sur au métaphorique ou inversement? », Verbum, 20, 4, pp. 405-434.

DOMINICY, Marc \& MARTIN, Fabienne, 2005, «A travers et au travers de: à travers / des emplois perceptuels aux emplois spatiaux », in Dendale, Patrick au travers de (éd.), 2005, Le mouvement dans la langue et la métalangue, Metz, Université de Metz, pp. 151-190. (Recherches Linguistiques, 27).

DOS SANTOS MACHADO, Liliane, 1999, « Les marqueurs entao, logo, alors alors / donc et donc: temporalité et causalité. Analyse contrastive portugais/français », Verbum, 4, pp. 469-484.

Ducrot, Oswald, 1970, «Peu et un peu », Cahiers de lexicologie, 16, 1, peu / un peu pp. 21-52.

DucroT, Oswald, 2002, "Quand peu et un peu semblent coorientés : peu après et un peu après ", Cahiers de linguistique française, 24, pp. 207-229.

DUTKA, Anna, 2002, « La mise à distance du discours cité en français et en polonais : la traduction des incises paraît-il et dit-on dans les textes littéraires », Faits de Langues, 19, pp. 99-108.

ELUERD, Roland, 1973, « Note sur appareil, engin et machine en français contemporain ", Cahiers de lexicologie, 22, pp. 59-67. peu après / un peu après paraît-il / dit-on

Engel, Dulcie \& Rossi-Gensane Nathalie, 2004, "Sémantique et fréquence : étude de c'est / il est dans un corpus journalistique », Revue romane, 39, 2, pp. 177-202.

ENGLEBERT, Annick, 1985, "L'opposition ne / ne...pas en ancien franne / ne...pas / çais ", Revue de linguistique romane, 49, 195-196, pp. 365-378.

ESKÉNAZI, André, 2005, «Occirre et tuer dans Vie de Saint Louis », Romanon / non pas nia, 123, pp. 273-291.

FALL, Khadiyatoulah \& GAGNON, Maurice, 1995, «Quelques connecteurs causals : comme-étant donné que-sous prétexte que », Langues et Linguistique, 21, pp. 69-89.

FÉRON, Corinne, 2005, «Modalisation et verbes d'opinion. Quelques remarques sur croire, cuidier et penser en usage direct dans La Queste Del Saint Graal », L'information grammaticale, 104, 1, pp. 15-21. 
FLAMENT-B OISTRANCourT, Danièle, 1992, « Un couple impossible : le couan / année ple an / année», in Bouchard, Robert e.a. (éds), 1992,Acquisition et enseignement / apprentissage des langues, Grenoble, LIDILEM, pp. 338-395.

FLAMENT-B OISTRANCOURT, Danièle, 1994, « Remarques sur un petit couple an / année rebelle : an / année », Langue française, 103, pp. 56-67.

FLAMENT-B OISTRANCOURT, Danièle, 1999, «Quelques aspects de ainsi et aussi consécutifs à la lumière d'un point de vue de non-francophone, Le gré des langues, 15, pp. 142-179.

FLOREA, Ligia-Stela, 1999, «En et dans. Syntaxe, interprétation, réféen / dans rence", in Amiot, Danye.a. (éds), 1999, Fonctions syntaxiques et rôles sémantiques, Arras, Artois Presses Université, pp. 27-37.

FLøTTUM, Kjersti, 2003, «À propos de quant à et en ce qui concerne », in Combettes, Bernard, Schnedecker, Catherine \& Theissen, Anne (éds), en ce qui concerne 2003, Ordre et distinction dans la langue et le discours, Paris, Champion, pp. 185-202.

FOURMENT BERNI-CANINI, Michele, 1986, «Anno : an / année, projet pour an / année une entrée de dictionnaire bilingue ", Lexicon philosophicum. Quaderni di terminologia filosofica e storia delle idee, 40, Rome, Edizioni dell'Atenco, pp. 9-15.

Fuchs, Catherine, 1988, «Encore, déjà, toujours : de l'aspect à la modali- encore / toujours té », in Tersis, Nicole \& Kihm, Alain (éds), 1988, Temps et aspects, Paris, Peeters/Selaf, pp. 135-148.

GAATONE, David, 1981, «Observations sur l'opposition très - beaucoup », Revue de linguistique romane, 45, pp. 74-95.

GAATONE, David, 1984, «Une allergie syntaxique en français. Réflexions sur l'opposition lui / à lui », Revue de linguistique romane , 48, 189190, pp. 123-140.

GAatone, David, 1991, "Les déterminants de la quantité peu élevée en français. Remarques sur les emplois de quelques et plusieurs », Revue romane, 26, 2, pp. 3-11.

GAATONE, David, 1991, « Note sur la pseudo-synonymie de Il s'agit et Il est question ", Cahiers de lexicologie, 58, pp. 81-91.

très / beaucoup AaTONE, David, 2006, « La problématique des notions d' " indéfinitude » et de " prédication » de quelques et de plusieurs », in Corblin, Francis, Fernando, Sylvie \& Kupferman, Lucien (éds), 2006, Indéfini et prédication, Paris, PUPS, pp. 25-35.

GARDNER-ChloRAS, Penelope, 1991, « Ni tu ni vous : principes et paradoxes dans l'emploi des pronoms d'allocution en français contemporain ", Journal of French Language Studies, 1, 2, pp. 139-155.

GONDRET, Pierre, 1976, "Quelques, plusieurs, certains, divers : étude sémantique », Le français moderne, 44, 2, pp. 143-152.

lui / à lui quelques / plusieurs

il s'agit / il est question quelques / plusieurs tu / vous quelques / plusieurs / certains / divers 
Gougenheim, Georges, 1970, «L'emploi des pronoms interrogatifs que et que / quoi quoi devant l'infinitif », in Deloffre, Frédéric e.a. (éds), 1970, Etudes de grammaire et de vocabulaire français, réunies sur l'initiative de ses collègues et amis pour son soixante-dixième anniversaire, $\mathrm{Pa}-$ ris, Picard, pp. 124-129.

Gougenheim, Georges, 1970, «Les prépositions en et dans dans les preen / dans mières œuvres de Ronsard ", in Deloffre, Frédéric e.a. (éds), 1970, Etudes de grammaire et de vocabulaire français, réunies sur l'initiative de ses collègues et amis pour son soixante-dixième anniversaire, Paris, Picard, pp. 66-76.

GougENHEIM, Georges, 1970, «Les pronoms interrogatifs : que et quo $i$ ", in Deloffre, Frédéric e.a. (éds), 1970, Etudes de grammaire et de vocabulaire français Réunies sur l'initiative de ses collègues et amis pour son soixante-dixième anniversaire, Paris, Picard, pp. 108-112.

GRÉA, Philippe, 2005, « Les locutions au milieu / centre de dans leur acception spatiale », Revue de sémantique et pragmatique, 18, pp. 87-111.

Guimier, Claude, 1978, «En et dans en français moderne. Etude sémantique et syntaxique ", Revue des langues romanes, 83, 2, pp. 277-306.

Guimier, Claude, 2000, "Non-congruence et congruence : alors que vs alors que / tandis que ", Syntaxe et sémantique, 1, pp. 39-78.

HADERMANN, Pascale, 1989, « La concurrence entre où et que après un antécédent exprimant une notion temporelle ", in Lorenzo, Ramón (éd.), 1997, Actas do XIX Congreso Internacional de Lingüistica e Filoloxía Románicas, Coruña, Pedro Barrié de la Maza, pp. 427-436.

HALmøY, Odile, 1979, « Remarques sur la distribution du couple an-année en français contemporain », Moderna Språk, 73, pp. 66-75.

HaLmøy, Odile, 1986, «Instant / moment : Un problème de synonymie lexicale », in Suomela-Härmä, Elina, Välikangas, Olli (éds), 1986,Actes du 9 ìme Congrès des Romanistes Scandinaves : Helsinki 13-17 août 1984, Helsinki, Société Néophilologique, pp. 81-96. (Mémoires de la Société Néophilologique de Helsinki, 44).

HaLmøy, Odile, 1995, “"Les mots ne sont pas des fromages” : la dichotomie saussurienne diachronie/synchronie et la distribution du couple an / année en français actuel », Langue française, 107, pp. 93-110.

HaLmøy, Odile, 2006, « De chose et d'autres. La série truc, machin, bidule. Éléments de distribution ", in Riegel, Martin e.a. (éds), 2006, tandis que au milieu de / au centre de que / quoi en / dans Aux carrefours du sens. Hommages offerts à Georges Kleiber pour son 60e anniversaire, Louvain, Peeters, pp. 511-529.

HAM0N, Sophie, 2004, « Propriétés syntaxiques et valeurs argumentatives des conjonctions parce que et puisque ", in Tenchea, Maria \& Tihu, Adina (éds), 2004, Prépositions et conjonctions de subordination, Timisoara, Excelsior art, pp. 145-158. 
Hanse, Joseph, 1973, «Car, comme, parce que, puisque », Bulletin de l'Académie Royale de Langue et Littérature Française, 51, pp. 195-225.

car / comme / parce que / puisque

Hasselrot, Bengt, 1973, "Période de dix ans : décade ou décennie? », décade / décennie in Martin, Robert \& Straka, Georges (éds), 1973, Mélanges de linguistique française et de philologie et littérature médiévales offerts à Monsieur Paul Imbs, Paris, Klincksieck, pp. 219-223.

IMBS, Paul, 1985, «Sur la concurrence de ainz et de mais en moyen franainz / mais çais », Verbum, 8, pp. 85-98.

IORDANSKAJA, Lidija \& MEL'CuK, Igor, 1995, «Traitement lexicographique en fait / en réalité de deux connecteurs textuels du français contemporain : en fait vs. en réalité ", ", in Shyldkrot, Hava Bat-Zeev \& Kupferman, Lucien (éds), 1995, Tendances récentes en linguistique française et générale. Volume dédié à David Gaatone, Amsterdam/Philadelphia, Benjamins, pp. 211-236.

IORDANSKAJA, Lidjia \& ARBATCHEWSKY-JUMARIE, Nadia, 2000, « Quatre prépositions causales du français : leur sémantisme et cooccurrence », Linguisticae Investigationes, 23, 1, pp. 115-159.

à cause de / sous l'effet de / de / par

JARREGA, Maria, 2002, «Étude de quatre adjectifs atypiques :pluriel, $m u l$ - pluriel / multiple / tiple, singulier et unique », Langue française, 136, 4, pp. 73-88.

JAYEZ, Jacques, 1982, "Quand bien même pourtant, pourtant quand même ; Concession et consécution dans le discours ", Cahiers de linguistique française, 4, pp. 189-217. singulier / unique quand bien même / pourtant / quand même

JAYEZ, Jacques, 1987, « Sémantique et approximation : Le cas de presque et à peine », Lingvisticae Investigationes, 11, 1, pp.157-196.

KaHLOUL, Mongi, 2004, "Tout compte fait, de toute façon : connecteurs conclusifs et/ou de clôture? ", Revue de sémantique et pragmatique, 15-16, pp. 235-252.

KATZ, Eva, 2002, «Systématique de la triade spatiale à, en, dans », Travaux de Linguistique, 44, pp. 35-49.

KLEIBER, Georges, 1978, «Sur l'emploi adversatif de mais et de ainz (ainçois) en ancien français ", Travaux de linguistique et de littérature, 16, 1, pp. 271-293.

KLEIN, Hans-Wilhelm, 1972, «"Moi” betonte form zu “je” ? Betrachtung presque / à peine tout compte fait / de toute façon zu einer neuen Darstellung des französischen Personalmorpheme ", Praxis des neusprachlichen Unterrichts, 19, pp.79-84. repris aussi dans : Hausmann, Franz Josef (éd.), 1983, Die französische sprache von beute, Darmstadt, Wissenschaftliche Buchgesellschaft, pp. 320-330.

KReUTZ, Philippe, 2003, «Tenter n'est pas essayer », Journal of French Language Studies 13, 3, pp. 301-322.

KuPFERMAN, Lucien, 1985, « Note sur dont / de qui / de quoi / duquel », Recherches linguistiques de Vincennes, 13, pp. 5-32.

à / en / dans mais / ainz / ainçois moi / je

dont / de qui / de quoi / duquel 
KWON-PaK, Song Nim, 2000, «Par terre vs sur le sol », Revue de sémantipar terre / que et pragmatique, 8, pp. 63-79.

KWON-PAK, Song Nim, 2006, "Entre vs parmi : deux prépositions au cenentre / parmi tre de la partition ", in Kleiber, Georges, Schnedecker, Catherine \& Theissen, Anne (éds), 2006, La relation partie-tout, Louvain/Paris, Peeters, pp. 651-667.

LAMIROY, Béatrice \& CHAROLLES, Michel, 2004, « Des adverbes aux connecteurs : simplement, seulement, malheureusement, heureusement », Travaux de linguistique, 49, pp. 57-79.

LAvieu, Belinda, 2004, " A l'aide de vs au moyen de : l'expression du moyen ", Revue de sémantique et pragmatique, 15/16, pp. 85-102.

LAVIS, George, 1973, « La concurrence entre penser, cuidier, croire chez Chrétien de Troyes ", in Marche Romane, Hommage au professeur Maurice Delbouille, 1973, Liège, Cahiers de l'A.R.U.Lg, pp. 147-168.

LAVIS, Georges, 1979, «L'étude de la synonymie verbale dans l'ancienne langue française. L'exemple de savoir et conoistre en moyen français », in Wilmet, Marc (éd.), 1979, Sémantique Lexicale et Sémantique Grammaticale en Moyen Français, Bruxelles, VUB Centrum voor Taal-en Literatuurwetenschap, pp. 97-128.

LÉARD, Jean-Marcel, 1986, «Ily a... qui et C'est... qui. La syntaxe comme compatibilité d'opérations sémantiques ", Lingvisticae Investigationes, 10,1 , pp. 85-130.

LEBAUD, Daniel, 1990/1991, «Savoir et connaître », Le gré des langues, 1 , pp. 165-179, et 2, pp. 182-190.

LEBSANFT, Franz, 1987, « Le problème du mélange du « tu » et du « vous » en ancien français ", Romania, 108, pp. 1-19.

LEEMAN, Danielle, 1989, « Est-ce qu'on peut aller au coiffeur ? ", Lingvisticae Investigationes, 13, 2, pp. 399-404.

LEEMAN, Danielle, 1992, « Remarques sur puisque et sur car », in Flament-Boistrancourt, Danièle (éd.), 1992, Travaux et recherches. Théories, données et pratiques en français langue étrangère, Lille, Presses universitaires de Lille, pp. 113-128.

LEEMAN, Danielle, 2002, «Me est un autre », in Anis, Jacques, Eskénazi, André \& Jeandillou, Jean-François (éds), 2002, Le signe et la lettre. En hommage à Michel Arrivé, Paris, L'Harmattan, pp. 349-358.

LEEMAN, Danielle, 2003, «Me et mo $i$ dans la complémentation verbale », in Haderman, Pascale, Van Slijcke, Ann \& Berré, Michel (éds), 2003, La syntaxe raisonnée. Mélanges de linguistique générale et française offerts à Annie Boone à l'occasion de son 60e anniversaire, Bruxelles, De Boeck/Duculot, pp. 151-166.

LEEMAN, Danielle, 2006, «Des jumeaux presque indistinguables : différents et divers", in Riegel, Martin e.a. (éds), 2006, Aux carrefours du sens. Hommages offerts à Georges Kleiber pour son 60e anniversaire, Louvain, Peeters, pp. 133-146. savoir / conoistre simplement / seulement à l'aide de / au moyen de

penser / cuidier / croire il y a... qui / c'est... qui savoir / connaître tu / vous à / au / chez puisque / car me / je / moi . 
LE GROUPE $\lambda-1,1975$, «Car, parce que, puisque», Revue romane, 10, pp. 248-280.

LE QUERLER, Nicole, 2006, " Les déterminants indéfinis tout, quelque, chaque, certain et la prédication de propriété ", in Corblin, Lucien, Ferrando, Sylvie \& Kupferman, Lucien (éds), 2006, Indéfini et prédication, Paris, PUPS, pp. 349-367.

LERAT, Pierre, 1972, « Le champ linguistique des verbes savoir et connâेtre », Cahiers de lexicologie, 10, 1, pp. 53-63.

Letoublon, Françoise, 1983, «Pourtant, cependant, quoique, bien que : dérivation des expressions de l'opposition et de la concession", Cahiers de linguistique française, 5, pp. 85-110.

LIEHR, Ursula, 1971, Jour-journée, an-année : Gestalt und Entstehung eines sprachlichen Strukturfeldes, Tübingen, Präzis. (Tübinger Beiträge zur Linguistik, 23).

LINDVALL, Lars, 1971, Sempres, lues, tost, vistes et leurs synonymes. Etude lexicographique d'un groupe de mots dans le français des XIIe-XVIe siècles, Stockholm, Almqvist \& Wiksell. (Romanica Gothoburgensia, 13).

LORENTZEN, Lise, 2006, " Le fonctionnement du pronom adverbial $y$ et la concurrence entre $y$, là et là-bas en emploi spatial ", Journal of French Language Studies, 16, 2, pp. 167-186.

MAILLARD, Michel, 1994, «Concurrence et complémentarité de $i l$ etça devant les prédicats impersonnels en français contemporain ou Comment distinguer une phrase asubjectale d'une phrase à sujet distinct », L'information grammaticale, 62, pp. 48-56.

Manguin, Jean-Luc, François, Jacques \& VictorRi, Bernard, 2005, « Polysémie adjectivale et rection nominale : quand gros et gras sont synonymes », in François, Jacques (éd.), 2005, L'adjectif en français et à travers les langues, Caen, Presses Universitaires de Caen, pp. 521-540.

MARQue-PuCheu, Christiane, 2000, «À mon avis et à mon goût : jugement de réalité et jugement de valeur ", in Englebert, Annick e.a. (éds), 2000, Actes du XXIIe Congrès International de Linguistique et de Philologie Romanes. Bruxelles, 23-29 juillet, volume VII, Tübingen, Niemeyer, pp. 459-472.

MARTIN, Fabienne \& DOMINICY, Marc, 2001, «A travers, au travers (de) et le point de vue ", Travaux de Linguistique, 42-43, pp. 211-227.

MARTIN, Robert, 1988, «Croire que $\mathrm{p} /$ penser que $\mathrm{p}$ », in Benezech, JeanLouis e.a. (éds), 1988, Hommage à Bernard Pottier, Paris, Klincksieck, pp. 547-555.

MARTIN, Robert, 1997, «An / année en moyen français », in Holtus, Günter, Kramer, Johannes \& Schweickard, Wolfgang (éds), 1997, Italica et Romanica : Festschrift fur Max Pfister zum 65. Geburtstag, Tübingen, Niemeyer, pp. 109-114.

car / parce que / puisque tout / quelque / chaque / certain

savoir / connaître pourtant / cependant / quoique / bien que jour / journée / an / année sempres / lues / tost / vistes y / là / là-bas il / ça gros / gras à mon avis / à mon goût

à travers / au travers (de) croire que / penser que an / année 
MeLleT, Sylvie \& MONTE, Michèle, 2005, «Néanmoins et toutefois : polyphonie ou dialogisme? », in Bres, Jacques e.a. (éds), 2005, Dialogisme et polyphonie. Approches linguistiques. Bruxelles, Duculot, pp. 249-263.

MERK, Georges, 1976, « Prière et oraison », in Kopp, Robert \& Doménech, Germán (éds), 1976, Mélanges offerts à Carl Theodor Gossen, Bern/Liège, Francke, pp. 595-634.

Meunier, André \& Morel, Mary-Annick, 1993, «Pas et point en français pas / point classique (Molière) », L'information grammaticale, 57, pp. 25-30.

MignON, Françoise, 2004, La phrase négative averbale : approche énonciative de non et pas dans des textes de théâtre, Thèse de doctorat, Toulouse, Université de Toulouse-Le Mirail.

Millet, Cindy, 2002, «Bien que etquoique : Deux manières différentes bien que / quoique d'exprimer la concession ", Cahiers AFLS (Association for French Language Studies), 8, 1, pp. 10-15.

MiÑONES, Laura \& SLEPOY, Silvia, 2004, Etude sur la connexité en français écrit : le cas de "sinon" et de "en fait, de fait" et "en réalité", München, Lincom.

MoIGNET, Gérard, 1965, «L'oppositionnon / ne en ancien français », Travaux de linguistique et de littérature, 3,1, pp. 41-65.

MOSEGAARD HANSEN, Maj-Britt, 2005, «A comparative study of the semantics and pragmatics of enfin and finalement, in synchrony and diachrony ", Journal of French Language Studies, 15, 2, pp. 153-171.

Muller, Claude, 1999, "Encore et toujours les modifieurs aspec- encore / toujours tuels; de encore à toujours", in Plenat, Marc e.a. (éds), 1999, L'emprise du sens : structures linguistiques et interprétations : mélanges de syntaxe et de sémantique offerts à Andrée Borillo par un groupe d'amis, de collègues et de disciples, Amsterdam, Rodopi, pp. 217-237.

Muller, Claude, 2003, "Les déterminants indéfinis négatifs aucun et pas un : portée, référence, interactions », Verbum, 25, 1, pp. 61-80.

NoAILlY, Michèle, 2006, «Moitié...moitié, etmi-...mi-..., son double», in non / ne en fait / en réalité non / pas toutefois 
PEETERS, Bert, 1993, «Commencer et se mettre à : une description axiocommencer logico-conceptuelle, Langue française, 98, pp. 24-48.

PÉRoz, Pierre, 2003, «Sur à propos de et la valeur d'à propos de sur. à propos de / sur Proximité sémantique et construction du sens ", in Combettes, Bernard, Schnedecker, Catherine \& Theissen, Anne (éds), 2003, Ordre et distinction dans la langue et le discours, Paris, Champion, pp. 409-423.

PICOCHE, Jacqueline, 1987, « La grâce et la merci [en ancien et en moyen grâce / merci français] ", Cahiers de lexicologie, 50, pp. 191-199.

PORHIEL, Sylvie, 2001, «Au sujet de et à propos de : Une analyse lexicographique, discursive et linguistique ", Travaux de linguistique, 42/43, pp. 171-181.

RABATEL, Alain, 2001, "Valeurs énonciative et représentative des « présentatifs »c'est, ily a, voici/voilà : effet point de vue et argumentativité indirecte du récit », Revue de sémantique et pragmatique, 9-10, pp. 43-74.

RÉMI-GIRAUD, Sylvianne, 1986, " Etude comparée du fonctionnement syntaxique et sémantique des verbes savoir et connaître ", in RémiGiraud, Sylvianne \& Le Guern, Michel, (éds), 1986, Sur le verbe, Lyon, Presses Universitaires de Lyon, pp. 169-306.

ReYLe, Uwe, 1998, "A note on enumerations and the semantics of puis and alors ", Cabiers de grammaire, 23, pp. 67-79.

RICKARD, Peter, 1964, «Toute jour, tout le jour, et toute la journée en français médiéval », Romania, 85, 2-3, pp. 145-180.

au sujet de /

à propos de c'est / il y a / voici / voilà

savoir / connaître toute la journée

RIVIÈRe, Nicole, 1992-1993, «Ily $a$ : il y a 10 ans, il y a 10 ans que, depuis il y a / il y a... que / 10 ans ", Modèles linguistiques, 14, 1, pp. 121-152. depuis

RodríGuez Somolinos, Amalia, 2000, «Mais, ains, ainçois en moyen français : syntaxe et sémantique ", Le moyen français, 46-47, pp. 449-467.

RODRÍGuEz SOMOLINos, Amalia, 1995, «Certes, voire : l'évolution sémantique de deux marqueurs assertifs de l'ancien français », Linx , 32, pp. 51-76.

ROEGIEST, Eugeen, 1982-1983, " "À" et "par" dans la construction factitive : l'EGLC dans la perspective de la linguistique contemporaine », Travaux de linguistique, 9-10, pp. 127-143.

Rosier, Laurence, 2002, « Sortes d'invectives : Approche syntactico-sémantique des termes espèce et sorte », in Lagorgette, Dominique \& Larrivée, Pierre (éds), 2002, Représentations du sens linguistique, München, Lincom Europa, pp. 137-146.

ROSSARI, Corinne, 1992, "De fait, en fait, en réalité : trois marqueurs en fait / en réalité aux emplois inclusifs ", Verbum, 14, 3, pp. 139-161. 
ROSSARI, Corinne, 1996, "Les marques de la consécution : propriétés communes et distinctives à la lumière de donc, de ce fait, et il en résulte que ", in Muller, Claude (éd.), 1996, Dépendance et intégration syntaxique. Subordination, coordination, connexion, Tübingen, Max Niemeyer, pp. 271-283.

Rouget, Christine, 1986, "Comment son et le sien mettent de l'ordre dans la syntaxe nominale », Recherches sur le français parlé, 8, pp. 105-117.

ROUGET, Christine, 1997, «Espèce de, genre de, sorte de à l'oral et à l'écrit », Recherches sur le français parlé, 14, 1, pp. 173-183.

RunNALls, Graham A., 1981, "'Mansion' and 'lieu' : two technical terms in medieval French staging ? , French Studies, XXXV, 4, pp. 385-393.

RuPPLI, Mireille, 1990, «L'opposition car / parce que ", L'information grammaticale, 46, pp. 22-25.

SAKARI, Ellen, 1997, «Observations sur quelques adverbes de temps (or, lors, alors) en moyen français », in Combettes, Bernard \& Monsonego, Simone (éds.), 1997, Le moyen français. Philologie et linguistique. Approches du texte et du discours, Paris, Didier Erudition, pp. 351-368.

SCHNEDECKER, Catherine, 2002, "Premier, principal, primordial: des adjectifs qui sortent du rang ? ", Langue française, 136, 4, pp. 89-103.

SCHNEDECKER, Catherine, 2005, «Autre vs. second : de vrais faux ordinaux », Cahiers de lexicologie, 86, 1, pp. 131-144.

SCHUMACHER, Nestor, 1971, "Evolution du vocabulaire des relations internationales : les adjectifs international, supranational, transnational, etc. et leurs équivalents allemands ", Le langage et l'homme, 15,1, pp. 48-58.

Soliman, Luciana T. \& SinI, Lorella, 2004, «L'alternance phorique ce / il c'est / il est dans la construction c'est / il est : étude contrastive avec l'italien ", Modèles linguistiques, 25, 1-2, pp. 347-360.

Straub, Sylvia, 1973-1974, "The Passive Prepositions De and Par ", de / par French Review, 47, 3, pp. 583-593.

TAmba, Irène, 1983, « Pourquoi dit-on "ton neveu, il est orgueilleux" et "ton neveu, c'est un orgueilleux" ? ", L'information grammaticale, 19 , pp. 3-9.

TAMBA, Irène, 1983, « La composante référentielle dans "un manteau de laine", "un manteau en laine” ", Langue française, 57, pp. 119-128.

Tovena, Lucia M. \& VAN PETEGHEM, Marleen, 2002, «Différent vs autre et l'opposition réciproque vs comparatif », Lingvisticae Investigationes, 25, 1, pp. 149-170. premier / principal / primordial

donc / de ce fait / il en résulte que

espèce de / genre de / sorte de mansion / lieu car / parce que or / lors / alors autre / second supranational / transnational / international 
VAN GRUNDERBEEK, Lieve, 2003, «Au-dessus de + contact : origines et au-dessus de / sur comparaison avec sur ", in Vanneste, Alex e.a. (éds), 2003, Mémoire en temps advenir. Hommage à Theo Venckeleer, Leuven/Paris, Peeters, pp. 485-501.

Van Peteghem, Marleen, 1992, " $I l$ vs $c e$ / ça en construction impersonil / ce / ça nelle », in Flament-Boistrancourt, Danièle (éd.), 1992, Travaux et recherches. Théories, données et pratiques en français langue étrangère. Colloque international organisé conjointement par l'Université Catholique de Lille (CLARIFE) et l'Université Charles-deGaulle - Lille III, Lille, Presses Universitaires de Lille, pp. 95-111.

VAN Peteghem, Marleen, 2001, «Autre vs différent : du pareil au autre / différent même? », in Amiot, Danny, De Mulder, Walter \& Flaux, Nelly (éds), 2001, Le syntagme nominal : syntaxe et sémantique, Arras, Artois Presses Université, pp. 141-160.

VANDELOISE, Claude, 1990, "Les frontières entre les prépositions sur et sur / à $\grave{a} »$, Cabiers de grammaire, 15, pp. 157-184.

VELAND, Reidar, 1998, Quand même et tout de même : concessivité, synonymie, évolution ", Revue romane, 33, 2, pp. 217-247.

Wilmet, Marc, 1992, «Sur les articles le / les génériques ou l'énigme du le / les cosmonaute ", in Hirschbühler, Paul \& Koerner, Konrad (éds), 1992, Romance languages and modern linguistic theory. Papers from the 20th linguistic symposium on romance languages Ottawa, 10-14 april 1990, Amsterdam / Philadelphia, Benjamins, pp. 373- 387.

Wimmer, Christine, 1994 \& 1995, «Si...que, d'autant plus que », L'information grammaticale, 63, pp. 32-34, et 64, pp. 9.

si... que / d'autant plus que

WinTHER, André, 1985, «Bon, (bien, très bien) : Ponctuation discursive et ponctuation métadiscursive », Langue française, 65, pp. 80-91.

ZÉNONE, Anna, 1982, "La consécution sans contradiction : donc, par conséquent, alors, ainsi, aussi »,Cahiers de linguistique française, 4, pp. 107-141.

ZÉNONE, Anna, 1983, "Concession et consécution dans le discours », $\mathrm{Ca}$ biers de linguistique française, 5, pp. 189-214. bon / bien / très bien donc / par conséquent / alors / ainsi / aussi donc par conséquent / alors / ainsi / aussi 


\section{Liste des expressions traitées dans les études réportoriées ci-dessus}

Voici les 273 unités et expressions qui font l'objet d'études répertoriées dans cette petite bibliographie. Elles figurent dans la seconde colonne du tableau. Le lecteur qui désire rechercher automatiquement les références qui correspondent à l'expression de son choix, peut se servir de la partie thématique de la bibliographie en ligne dont l'adresse Internet figure en note 2 .

à, à cause de, à l'aide de, à l'occasion de, à lui, à mes yeux, à mon avis, à mon goût, à nouveau, à partir de, à peine, à propos, à propos de, à travers, ainçois, ains, ainz, ainsi, ainsi que, airelle, alors, alors que, an, année, apparaître, appareil, au, au centre de, au contraire, au fait, au milieu de, au moment où, au moyen de, au sujet de, au travers de, aucun, au-dessus de, aussi, autre, avec, avoir peur, beaucoup, bidule, bien, bien que, bon, bout, bribe, brin, c'est, c'est quoi, ça, car, ce, cependant, certain, certains, certes, c'est, c'est... qui, chaque, chez, chose, comme, commencer, connaître, conoistre, contre, côté, craindre, croire, cuidier, d'accord, dans, dans la réalité, dans une heure, d'autant plus que, de, de ce fait, de même que, de nouveau, de qui, de quoi, de toute façon, décade, décennie, décider, dedans, défendre, autoriser, depuis, différent, différents, dit-on, divers, donc, dont, duquel, durant, effectivement, en, en ce qui concerne, en effet, en fait, en réalité, en revanche, encore, encore que, enfin, engin, entre, espèce, essayer, étant donné que, faire, finalement, forcément, genre de, grâce, gras, gros, $i$, il, il en résulte que, il est, il est question, il paraît que, il s'agit, il semble que, il y a, il y a... que, il y a...qui, instant, interdire, international, je, jour, journée, là, là-bas, laisser, le, le sien, les, lieu, longtemps, longuement, lors de, lues, lui, machin, machine, mais, mansion, me, même si, merci, mi-, moi, moitié, moment, mourir, multiple, myrtille, ne, ne...pas, néanmoins, nécessairement, n'importe quel, non, non pas, occirre, on, or, oraison, où, oui, par, par conséquent, par contre, par terre, paraît-il, paraître, parce que, parmi, particulièrement, pas, pas un, pendant, penser, périr, permettre, petite voiture, peu, peu après, pluriel, plusieurs, point, pour, pour ce qui est de, pour moi, pourtant, premier, prendre une décision, presque, prière, primordial, principal, puis, puisque, qu'est-ce que c'est, quand bien même, quand même, quant à, que, quelque, quelques, question, quoi, quoique, redouter, réellement, rien de moins que, rien moins que, savoir, se mettre à, second, selon moi, sembler, sempres, seulement, si m'aïst dex, se dex m'aüt, si...que, simplement, singulier, soit, son, sorte de, sous l'effet de, sous prétexte que, supranational, sur, sur le sol, surtout, tandis que, tenter, tost, toujours, tout, tout compte fait, tout de même, tout le jour, toute jour, toute la journée, toutefois, transnational, très, très bien, truc, tu, tuer, un peu, un peu après, une heure plus tard, unique, uniquement, vistes, voici, voilà, voire, voiturette, vous, $y$. 